\title{
GLOSSARY OF TEXTILE TERMS
}

Batik An Indonesian word commonly used in Europe and English-speaking countries to describe resist dyeing where a resist medium (usually molten wax) is applied to woven cloth by means of special tools, brushes or stamps.

Block-printing Printing dyes, mordants or a resist medium (such as gum) onto a textile by means of relief-carved wooden block (a different block for each colour). In India, the blocks are usually 23 or 30 centimetres (9 or 12 inches) square in size.

Brocade Figured textiles with the patterning woven in supplementary, usually discontinuous, weft threads.

Brocaded A pattern made by wefts which only go to the width of the motif and are then turned back.

Calico A plain weave, opaque cotton fabric.

Cocoon The hardened pupa case of moths and butterflies, here referring to that created by silkworms. It consists of hundreds of metres of continuous filament (silk) which can be reeled from the cocoon after boiling.

Complementary threads/elements Threads usually of contrasting colours woven into a textile to create a pattern. Unlike supplementary threads, they are structurally integral parts of the weave, and their removal damages and weakens the fabric.

Continuous threads Those threads that extend the full length or width of the textile.

Cords Refers to the threads of the figure harness on the drawloom which control the pattern in the width of the fabric. The greater the number, the finer and more elaborate could be the design.

Count, thread The number of warp and weft threads found in a specified linear measurement, such as a centimetre or inch. A high thread count usually denotes fine, thin threads (or else medium-sized threads in a very dense weave). Thus a 200-count cotton has 100 warp and 100 wefts per inch (39 warp/39 weft per centimetre).

Cutwork Warp or weft floats (at the back of the textile) cut away by hand, so that the pattern may appear as if woven in discontinuous supplementaryweft technique (a more time-consuming process).

Damask A weave and, by extension, a fabric which was usually (but not essentially) self-coloured. The pattern is created by the contrast in the reflection of light from the warp to the weft - the vertical and horizontal elements. The meaning is unchanged since the eighteenth century.

Discontinuous weft A weft thread that does not extend the full width of the textile. Usually of a contrasting colour to the ground threads (if supplementary) or to surrounding weft threads (if tapestry-woven).

Drawloom Believed to be of Sassanian Persian origin (c. AD 200). The fully developed drawloom uses a double harness set, one providing the weave,

$$
\text { [ xii ] }
$$


the other the figured patterning. Drawlooms developed differently in the Far East, Middle East and Europe, until the early nineteenth century invention of the Jacquard loom, most drawlooms required 'drawboys' who activated the pattern harness by lifting sets of threads. Today, Indian figured textiles woven commercially are usually created on Jacquard looms.

Figured fabric A textile in which patterning is woven into the cloth rather than painted, printed, dyed or embroidered. Figured textiles are popular, but erroneously, called 'brocades'. They include those fabrics with continuous or discontinuous supplementary warp and weft patterning; sammitas and lampas, velvets, etc. Historically figured fabrics were woven using traditional drawlooms (naksha, jala); today Jacquard attachments are generally used.

Filament A continuous fibre created by natural (e.g. silk) or man-made processes. In silk cocoons, one or two filaments are created by the caterpillar to form the cocoon; in synthetic fibres the filament is created by being extruded through a 'spinneret'.

Gauze In true gauze weaves the warps are crossed and uncrossed between the wefts at intervals to create a transparent openwork fabric. The name is also given to plain-woven fabrics where the warps do not cross, but are often 'paired', creating uneven spacing in the weave between the series of warp threads. (This makes the textile look more transparent than it otherwise would be).

Jacquard A punch-card pattern-selecting device for handlooms or powerlooms, which was originally invented to replace the drawboy. It was refined and patented by J.M. Jacquard in 1804. Its speed and ease of use has made the older drawlooms obsolete throughout most of India.

Lampas A figured textile that has patterning created through at least two different warps and wefts in the weaving. Warp-faced and weft-faced integrated weave structures form the motifs, typically polychrome, using plain weaves and satins.

Lustring A lightweight crisp silk popular in the seventeenth and eighteenth century with a lustre given by preliminary treatment of the warp.

Mordant A colourless chemical (metallic oxide) that is necessary to bind dye to a cellulose fibre such as cotton or linen. The two most common mordants in India are (1) aluminium sulphate (alum), which binds, for instance, alizarin dyes to cotton and produces bright colours; and (2) ironbased compounds which in alizarin create the darker hues.

Muslin Fine, sheer, often transparent cotton fabric. Usually has high thread counts, ranging from about 150 to 300 .

Organzine Undegummed, twisted silk warp threads used to create organza. The silk filling yarn (wefts) used with organzine, called tram, is not so highly twisted.

Plain weave An over-one, under-one weave structure. Also called tabby weave.

Powerloom A loom powered by steam or electricity, making it much faster to use than a handloom.

Raw silk Undegummed silk; where the sericin has not been removed from the filaments. 
Reel Process by which silk filaments, in groups of six or more, are unwound from their cocoons and wound onto a circular contraption, which is also called a reel.

Resist dyeing Any form of dyeing where the dyestuff is prevented from adhering to selected areas of the thread or woven textile.

Satin A weave, and by extension, a fabric with a smooth surface in which the warp threads cover the wefts completely.

Selvage The outer edge of a textile parallel to the warp. It is made by the weft threads wrapping round the outermost warp threads. Often the group of warps threads at the selvage are more densely set than the rest of the fabric, making the selvage stronger than the inner cloth.

Shafts 'Foot-figured' silks were made without the drawloom mechanism. The warp was entered on the heddles of a series of wooden battens which were operated by treadles to give simple geometric designs.

Shuttles The weaver creates the textile by lifting alternate warp threads and passing the shuttle containing the weft from side to side of the loom. Tiny shuttles were used for brocading (see above). The designer had to let the weaver know how many shuttles would be needed in each line of the design - to work out the cost.

Silk Natural fibre produced by silkworms. Originally cultivated by the Chinese about 2500 BC. Many filaments are used to create a single fine thread, which, when woven, creates a thin, lustrous fabric.

Silkworm The caterpillars of moths of Bombidiceae and Saturnidae families, from which silks are derived.

Spin/spun Fibres of limited length (e.g. cotton, wool, linen) can be carded (aligned so the fibres lie parallel to each other) and then drawn out and twisted to form thread. The process is called spinning.

Supplementary warp or weft Supplementary threads are those added to a textile that already has one set of warp and weft threads. If supplementary threads are removed from a woven textile, the remaining fabric will still be complete.

Tie-dye Called bandhani (G) in western India. Resist patterning created on an already-woven textile by tying selected sections of cloth with thread, so preventing the dye from entering the tied areas.

Tissue The generic name in England for a silk with two warp and two weft systems. There were many varieties. A single tissue had one pattern weft from selvage to selvage and thus had one colour in the pattern, a double tissue had two colours, etc. Tissues could, however, be brocaded and still be tissues. The French term for this, lampas, has not changed in meaning.

Twill A weave and, by extension, a fabric, in which the warp and weft interlace at one or more removes as the textile progresses. It was a supple material used for linings and softly draped fabrics.

Warp Set of parallel threads mounted on a loom frame, kept in supply on a warp beam. In India, handweavers make warps long enough to make three to six saris at a time. (The length of the warp determines the overall length of the woven cloth coming off the loom.) 
Warp-faced weave A cloth wherein the warp threads predominate on the face of the fabric. In terms of fabric count, this can also result from there being either considerably more warp threads than weft, or else much thicker warp threads than weft.

Weft A set of threads that runs at right angles to the warp, interworking with them to create various structures of weaves, such as plain or twill weave.

Weft-faced weave A cloth wherein the weft threads predominate on the face of the fabric. In terms of fabric count, this is produced by there being either more weft threads than warp, or else thicker weft threads than warp.

Whitework Embroidery using white threads upon a white fabric, which is usually fine and translucent so the embroidery will stand out.

Wild silk Silk from the products of silkworms that are not varieties of Bombyx mori. Because their filaments are flat and spiral instead of circular or triangular, and their sericin is difficult to remove, they usually cannot be mechanically reeled or woven on powerlooms. Sometimes the spun threads from the broken ends and 'floss' from $B$. mori cocoons are also - erroneously - called wild silk instead of raw silk. Indian wild silks include tasar, muga and eria (endi).

Yarn-dyeing The yarn used to weave a textile is dyed before it is woven (as warp or weft). 